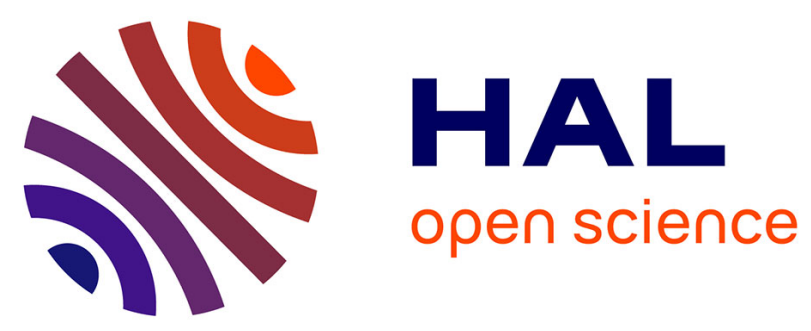

\title{
BLPAD.Core: A Multi-Functions Optimizer Towards Daily Planning Generation in Home Health Care
}

\author{
Liwen Zhang, Elyes Lamine, Franck Fontanili, Christophe Bortolaso, \\ Marianne Sargent, Mustapha Derras, Hervé Pingaud
}

\section{- To cite this version:}

Liwen Zhang, Elyes Lamine, Franck Fontanili, Christophe Bortolaso, Marianne Sargent, et al.. BLPAD.Core: A Multi-Functions Optimizer Towards Daily Planning Generation in Home Health Care. AICCSA 2020 - 17th International Conference on Computer Systems and Applications, Nov 2020, Antalya (virtual), Turkey. 6 p., 10.1109/AICCSA50499.2020.9316522 . hal-03109972

\section{HAL Id: hal-03109972 https://imt-mines-albi.hal.science/hal-03109972}

Submitted on 1 Mar 2021

HAL is a multi-disciplinary open access archive for the deposit and dissemination of scientific research documents, whether they are published or not. The documents may come from teaching and research institutions in France or abroad, or from public or private research centers.
L'archive ouverte pluridisciplinaire HAL, est destinée au dépôt et à la diffusion de documents scientifiques de niveau recherche, publiés ou non, émanant des établissements d'enseignement et de recherche français ou étrangers, des laboratoires publics ou privés. 


\section{BLPAD.Core: a Multi-functions Optimizer towards Daily Planning Generation in Home Health Care}

\author{
Liwen Zhang \\ DRI and Industrial Engineering Centre \\ Berger-Levrault and IMT Mines Albi \\ Labège, France and Albi, France \\ liwen.zhang@berger-levrault.com
}

\author{
Christophe Bortolaso, Marianne \\ Sargent \\ Berger-Levrault \\ Labège, France \\ \{first name.last name\}@berger- \\ levrault.com
}

\author{
Elyes Lamine \\ Industrial Engineering Centre \\ University of Toulouse-IMT Mines Albi \\ Albi, France \\ elyes.lamine@univ-jfc.fr
}

\author{
Mustapha Derras \\ Berger-Levrault \\ Labège, France \\ mustapha.derras@berger-levrault.com
}

\author{
Franck Fontanili \\ Industrial Engineering Centre \\ University of Toulouse-IMT Mines Albi \\ Albi, France \\ franck.fontanili@mines-albi.fr
}

\author{
Hervé Pingaud \\ CNRS LGC \\ University of Toulouse-INU \\ Champollion \\ Albi, France \\ herve.pingaud@univ-jfc.fr
}

\begin{abstract}
Today, a majority of the elderly want to live longer in autonomy and comfort. Since there is not enough space available in specialized institutions, Home Health Care Services (HHCS) constitute an important addition that reinforces the traditional system. With the increase in HHCS demands, the main challenge in this distributed system is the organization of care services in an HHC institution. This organizational problem is widely studied as a Home Health Care Scheduling and Routing Problem (HHCSRP) in the Operations Research (OR) community. Facing the diversity and complexity of demands, as done in OR, the formulated solution-oriented model aims at providing decision making support for the decision maker in HHC systems. This leads obstacles in the dataset / benchmark based experimental environment and critical analysis such as the degree of the optimality regarding generated solutions and the comprehensive visualization of solutions. In this paper, based on a systemic analysis of the existing benchmark and the solution approach for HHCSRP, a prototype named BLPAD.Core is introduced. It provides a full decision support system to the decision-maker in the HHC system. The functionality consists of an instance generation based on the real dataset, operational planning generation and solution visualization. This research highlights the scientific challenges and attempts to respond to the complicated expectations of HHC systems in a comprehensive manner.
\end{abstract}

Keywords-BLPAD, Home Health Care, Use case generation, Benchmark, Optimization, Planning Visualization

\section{INTRODUCTION}

Due to the rapid development of public health and medical science and technology, various acute infectious diseases have been controlled, the average life expectancy has been greatly extended, the population is rapidly aging. Also, chronic infectious diseases have become the main factor endangering people's health. These diseases have multiple etiologies and complex pathogenesis and are often accompanied by many psychosocial problems. There is no specific radical cure. Once an individual is sick, he will need continuous medical services for life. For these diseases, preventive health care and treatment such as medicine, psychological and social services are indispensable. This has caused significant changes in the quality and quantity of society's demand for medical and health services. On the other hand, the highly specialized medical service system has been difficult to adapt to the needs of social health care services. Its main disadvantage is that it focusses on physiological and biological causes and ignores the impact of psychosocial factors on health and disease. Emphasis on the diagnosis and treatment of diseases, neglecting the prevention and health care is limited to hospital services, unable to meet the rapidly growing social needs.

The aging population and a medical system which focuses on treatment rather than prevention contributes in a rapid growth of medical expenses. This leads to the unreasonable distribution and great waste of medical resources. The sharp increase of medical expenses has brought an increasingly heavy burden to the country and its enterprises and has affected the development of our society and economy. How to use the limited health resources to control the growth of medical expenses and improve the health level of the whole population has become a worldwide research question. Home Health Care Services (HHCS) in this respect is an important reason for its rapid development. The main challenge in this distributed system is the organization of the care services in an HHC structure. Guaranteeing the good coordination between the caregivers and the care beneficiaries will facilitate the planning of the care services to achieve business objectives while respecting the constraints inherent in an operational field. This issue is often addressed as a problem in optimizing practitioners' schedules, a subject that is widely studied by the Operations Research (OR) community. It is described in the literature under the heading Home Health Care Scheduling and Routing Problem (HHCSRP) [1] [2]. Without going into a detailed analysis of these studies, we were able to identify sources of diversity in this available research. In addition, the HHCSRP model can also be declined in a large number of variants depending on the HHC business constraints that need to be satisfied, and it would be inappropriate to assume that it is a static list. The rise of activity in the operational field will inevitably reveal new characteristics. Facing this diversity, the solution-oriented mathematical models that are trying to find the best operational coordination solution by using the OR approach are various as well. When the mathematical model is well built in conformity to the requirements specified by an HHC structure, it is difficult to find a real dataset that with $100 \%$ confirmation to the assumptions, the objectives function and the constraints modelled in our mathematical formulation. Consequently, this will lead to another obstacle regarding the solution evaluation and the determination of the optimality of the generated solutions, as well as the solution visualization for providing a systemic decision-making support to the deciders in the HHC structure.

In this respect, there are three primary aims of this research work: 1) To investigate the existing benchmark among the literature, then establish a standard in conjunction with the 
special parameters regarding our formulated mathematical model. 2) To ascertain the most adapted algorithms in the HHCSRP, for the purpose of solving our formulation by the different algorithms through a comparative evaluation platform and 3) to provide a decision-making visualizer to visualize the solution in a comprehensive manner in order to help the decision-maker doing the critical analysis regarding the generated solution.

The content of the remaining sections is structured as follows. Firstly, we will review the previous research works about the existing benchmark and the solution approach for the HHCSRP. Next, the 3 modules of BLPAD.Core are introduced to respond to the formulated HHC operational coordination requirement. The detail software structure and the data structure with respect to input/output of each module, will be presented, respectively. Finally, we conclude this work and address the outlook.

\section{STATE OF THE ART}

\section{A. Solution approch of HHCSRP}

Studies over the past two decades have many approaches to solve the HHCSRP addressed previously. Normally the approach is categorized into two groups : 1) the exact method which is trying to find the optimum solution concerning the formulated mathematical model based on Integer Linear Programming (ILP) or Mixed Integer Linear Programming (MILP), in spite of the computing time. For example, a human resource's synchronization problem [3] [4], a lunch break requirement [5], a care plans satisfaction problem [6] and a multiples treatments problem [7] are formulate under MILP. This approach is frequently solved by the commercial mathematic such as CPLEX, Gurobi, LocalSolver. 2) the approximate method is used to find an approximate solution to solve optimization problems. Based on the metaheuristic or algorithm, this approach can usually derive a solution with quality assurance in a reasonable computing time. In light of the efficiency of the algorithm in order to evaluate the solution generated under the different approaches, we opt for the approximate method as our solution approach developed in BLPAD.Core. Among the recent research works, Decerle et al. [8] introduced a memetic algorithm involving two original crossing operators to address HHCSRP with time windows and synchronization constraints at the care services level. A harmony search algorithm is presented by Lin et al.[9]. to handle jointly the rostering, routing and re-rostering for $\mathrm{HHC}$ services. The algorithm Tabu Search (TS) is largely used for solving the HHCSRP, Hertz and Lahrichi [10] consider the deterministic patients to caregivers assignment for $\mathrm{HHC}$ service by using TS. A daily planning generation using public transport [11], a periodic home health care logistics [12] [13], a sustainable delivery scheduling [14], a robust planning model considering the temporal uncertainty [15], a real-time scheduling problem with the disruption management [16] are solved by TS with the good performance. Moreover, Simulated Annealing (SA) is widely used for approximate solution generation in HHCSRP, such as an interdependent services based scheduling problem [17], a bi-objective green routing problem [18], a simultaneous delivery and pick-up problem [19], a workforce scheduling problem [20], a multimodal scheduling problem [21]. This is performed very well by SA in terms of computing time and gap study in comparison with the optimum objective function value. This literature review set out to investigate the usefulness and the efficiency of SA and TS as our target metaheuristic solutions approaches embedded in our optimizer of BLPAD.Core. The choice of algorithmic parameters of TS and SA is studied by [22] and [23].

\section{B. Benchmark study}

According to our review, there are 5 accessible benchmarks among the literature addressing the HHCSRP recently. They all aim to solve the HHCSRP with time windows but taking into consideration the different business constraints. The benchmark proposed by Decerle et al. [24] consists of 10 instances, the instance characterized by the number of visits, the number of care staff members, the number of synchronized visits, the average distance between the visits and the average time window size for each category. The second is introduced by Decerle et al. [8], including 7 instances of which the last 3 are the combinations of the 4 previous instances. The instance is characterized by the number of care services to be performed by a nurse or by an auxiliary nurse, the number of available nurses and auxiliary nurses, the number of home health care offices, the number of synchronized visits and the average distance between the visits. The third is published by Mankowska et al. [25], including 7 groups with 70 instances total (each group has 10 instances, randomly generated), characterized by number of patients, number of patients who request single service, number of patients who request double service and number of caregivers. The fourth is presented by Braekers et al. [18] including 90 instances : 30 small ones (10-25 services) and 60 realistic size ones (50-300 services). They are characterized by number of full-time nurses (8-10 hours/day), number of part-time nurses (4-6 hours/day), number of care services, number of patients and the transportation mode (car, public or mixed). At last, Hiermann et al. [21] presents a benchmark with 4 large size instances. They are characterized by number of caregivers, number of services, number of patients and the detail characteristics for each element such as qualification of caregivers, qualifications required for services.

Our contribution in this paper is resumed in 3 essential points. 1) Based on the benchmark [21] which is similar to our problem, we can define the standard format of our use case for feeding our optimizer in BLPAD.Core, combined with the special parameters required regarding our mathematical formulation presented in our recent research work [26]. 2) Two powerful metaheuristics (TS and SA) are ready to find a daily HHCSRP solution. 3) A comprehensive visualizer aims at providing the decision-making supports for the decision maker.

\section{BLPAD.CORE: HHCRSP CASES GENERATOR, SOLUTIONS OPTIMIZER AND VISUALIZER}

BLPAD [27], the previous version of BLPAD.Core, included Simulated Annealing (SA) algorithm to generate an optimized solution. However, we lacked a reference to evaluate the quality of the solutions obtained at the end of BLPAD. Moreover, BLPAD is not capable performing a complete experiment based on the real dataset. We have therefore launched an extensive study on this particularly important point relating to the proposal of a set of decisions that would make the operational coordination of the HHC visible. Fig. 1 schematizes the 3 modules of BLPAD.Core, as well as the information flow between these modules.

The interest of the BLPAD.Core is to create a software environment suitable to addressee the real cases in HHCSRP. The prototype of BLPAD.Core is composed of 3 modules 


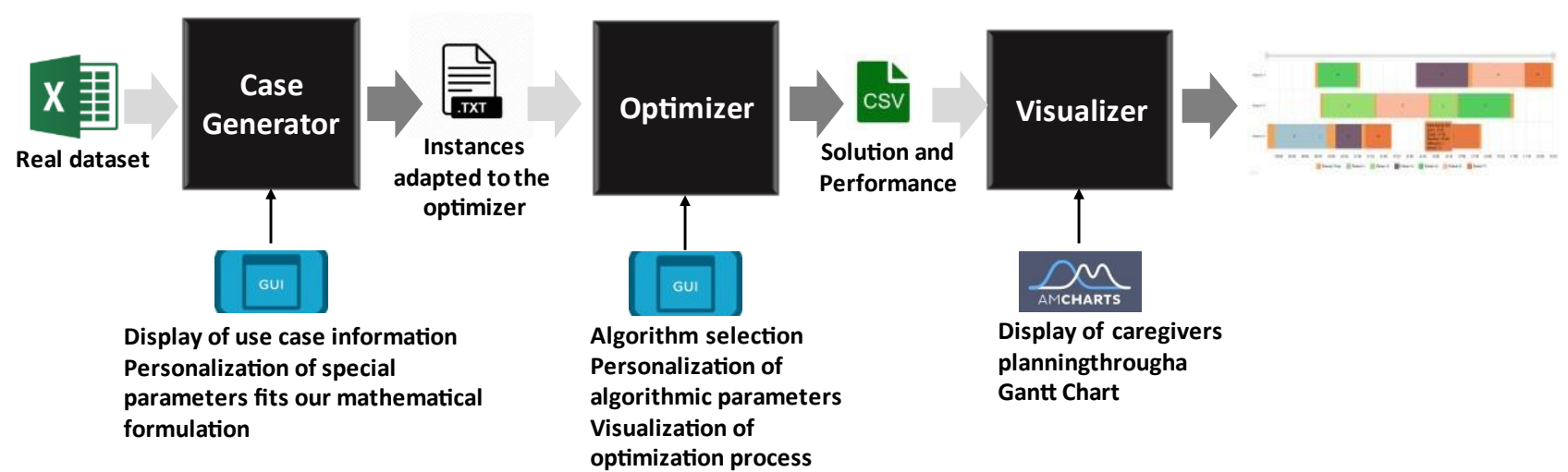

Fig. 1 Architecture of the research prototype BLPAD.Core

(case generator, optimizer, visualizer) with the successive objectives of :

- Generating instances adapted to the metaheuristic-oriented mathematical model presented in [26]. The data structure is inspired by the similar benchmark in the literature, based on real dataset in operational field.

- Solution optimization by using 2 metaheuristic algorithms based on our metaheuristic-oriented mathematical model, taking the instance generated by the generator. Thus, we are able to perform the comparative study and critical analysis with regard to the solutions generated by the different algorithms but under the same formulation.

- Visualization of these solutions in a comprehensible form to enable the quality assessment and the drawing of conclusions on the comparison exercise.

\section{A. Case generator}

This generator makes it possible to transform data provided by the real dataset into structured data in a .txt format, respecting the format provided by an HHCSRP benchmark published quite recently [21]. The output file of this generator can then feed our optimizer in order to find an optimum operational planning solution.

\section{BLPAD Core Generator}

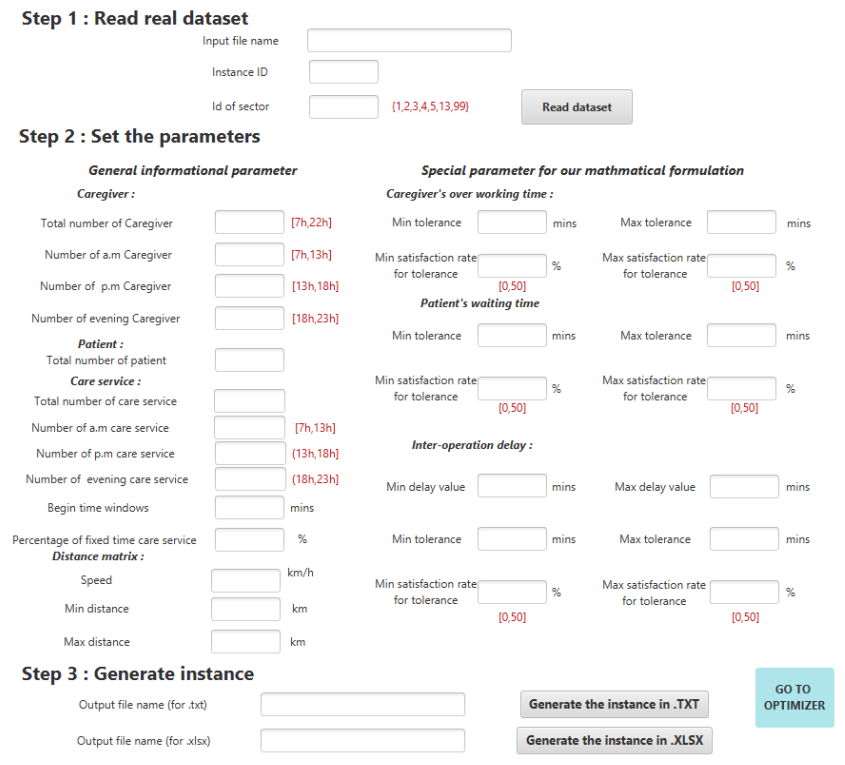

Fig. 2 Graphical interface of BLPAD.Core - Case generator
Fig. 3 is a metamodel of the input and output knowledge of case generator. The data is first grouped according to the geographic areas of each care service. Then, users can choose in which geographic area(s) they want to carry out the planning. According to this study configuration, the interface (Fig. 2) will display the key information from the real dataset that characterizes the use case. The general information, such as the total number of active caregivers, the total number of care services to be performed, the total number of patients, is displayed in "general informational parameter" section. The displayed care services are also grouped by the period of a day in order to observe their temporal distribution and then facilitate the definition of indicators to identify the difficulty of each instance. Some explanations shown in red for giving some detail information, for example the request in the morning (a.m.) is from 7:00am to 1:00pm. As the real dataset is not sufficient by itself to feed the optimizer (e.g. lack of the distance matrix or time windows for each care service), the interface provides options for users to complete them in order to meet the requirements of our formulation developed in optimizer. In the "Special parameters for our formulation" section, the given option is to complete our instance to fit our own formulation for characterizing caregivers' and patients' satisfaction. These special parameters are customizable by users, some of these fields are restricted, which is indicated below the corresponding field, written in red. The result is finally generated pseudo-randomly between this given maximum and minimum value. Having completed all of these intelligence operations, the case generator finally delivers a particular instance of a scheduling problem, partly based on the real dataset from the operational field.

\section{B. Optimizer}

This optimizer embeds a metaheuristic-oriented mathematical model and a solver with the two selected metaheuristics (Simulated Annealing (SA), Tabu Search (TS)), developed in Java. The solver aims at generating an optimum HHCSRP daily solution that formulated by the mathematical model. The description of the mathematical model such as data declaration, retained assumptions, considered constraints and optimization criteria were presented in our research work [26]. Fig. 3 presents a metamodel of the input and output knowledge of Optimizer.

The interface of the optimizer enables the choice of a metaheuristic among the 2 available options, with the input of the corresponding algorithmic parameters (Fig. 4). As for the parameters for each metaheuristic embedded in the optimizer, we present them based on the study by [28], which has drawn 


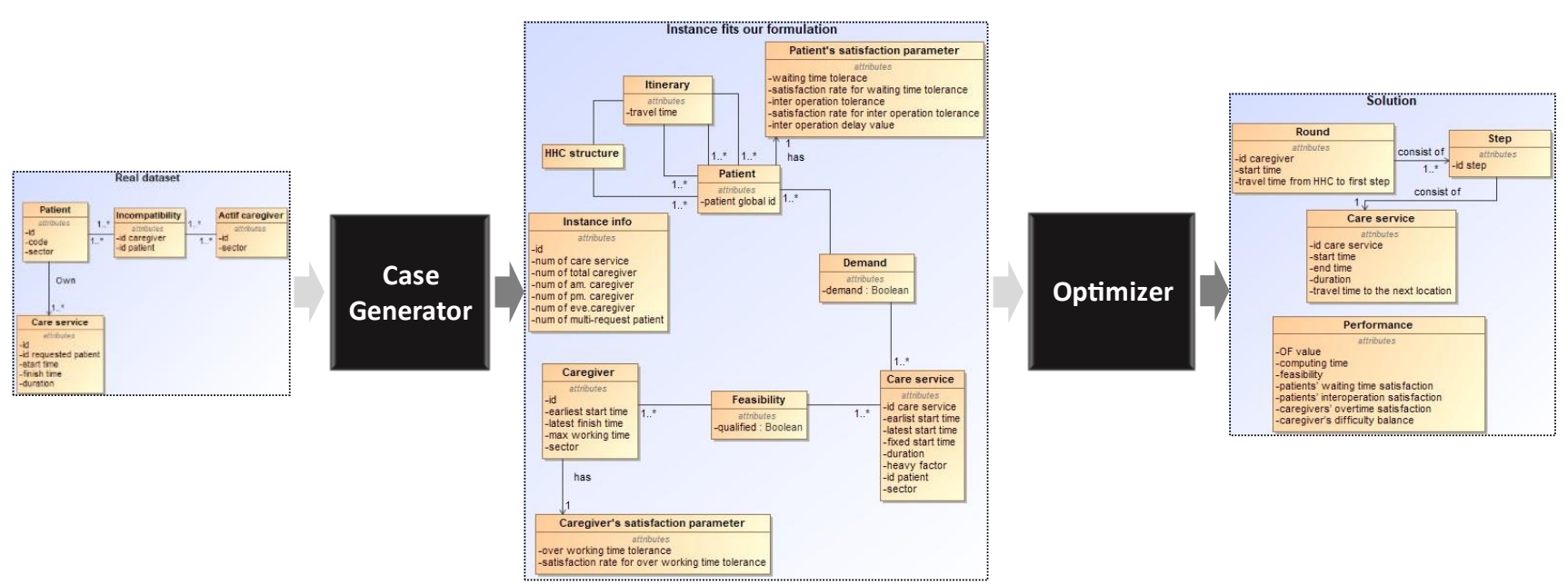

Fig. 3 BLPAD.Core input and output knowledge metamodel - Case generator and Optimizer

up a list of such parameters for the most popular metaheuristics (Table I).

The optimizer starts its operations by reading an instance generated by the case generator and then needs an initial point in the decision variable's space. This will be the starting point for searching a solution. The rule for this initialization is very simple: all the care services to be executed are assigned to all the caregivers according to a distribution called the randomly average distribution. For example, for a use case including 100 care services and 10 caregivers, the initial solution leads to 10 care services picked randomly in 100 care service per caregiver.

TABLE I STANDARD PARAMETERS OF THE METAHEURISTICS DEVELOPED IN THE BLPAD.CORE - OPTIMIZER

\begin{tabular}{|c|c|c|}
\hline Metaheuristic & Algorithmic parameter & $\begin{array}{l}\text { BLPAD.Core } \\
\text { - Optimizer }\end{array}$ \\
\hline \multirow{2}{*}{ Ant Colony } & Pheromone evaporation & \\
\hline & Pheromone weighting & \\
\hline \multirow{3}{*}{ Genetic Algorithm } & Crossover Probability & \\
\hline & Mutation Probability & \\
\hline & Population size & \\
\hline \multirow{4}{*}{ Harmony Search } & Distance Bandwidth & \\
\hline & Memory Size & \\
\hline & Pitch Adjustment Rate & \\
\hline & $\begin{array}{l}\text { Rate of Choosing from } \\
\text { Memory }\end{array}$ & \\
\hline \multirow{2}{*}{$\begin{array}{l}\text { Simulated } \\
\text { Annealing }\end{array}$} & Annealing rate & \multirow{2}{*}{$\mathbf{X}$} \\
\hline & Initial temperature & \\
\hline Tabu Search & Tabu list length & $\mathbf{X}$ \\
\hline $\begin{array}{c}\text { Variable } \\
\text { Neighborhood } \\
\text { Search } \\
\end{array}$ & N/A & \\
\hline
\end{tabular}

The interface also allows to enter the number of neighborhood solutions to be created during an optimization cycle (one iteration). The different possible "exchanges" to compose a new order of care services in the current neighborhood. These are operations to be performed on an ordered list of care services, for example changing the scheduling of care services in a round, or even changing the round to which a care service is assigned. The stop criteria of the algorithms is controlled by a maximum number of iterations set by the user, with the exception of SA, which will be stopped when a minimum required temperature is reached.

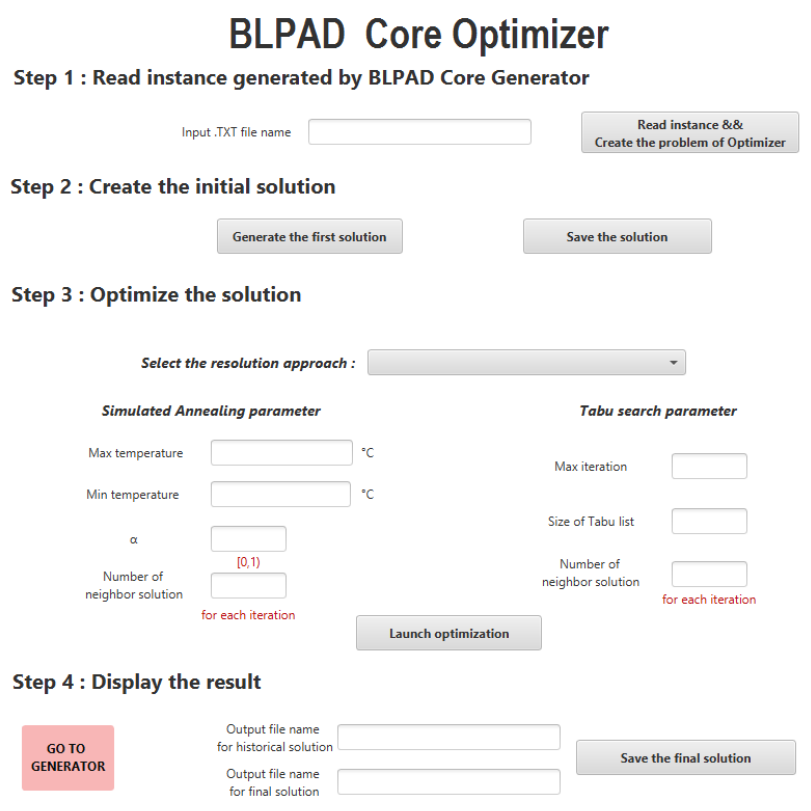

Fig. 4 Graphical interface of BLPAD.Core - Optimizer

When the optimization is finished, the optimizer generates 2 output files in .csv format:

- The first output contains the planning solution with each line the performed care services in theirs daily round, characterized by the performed caregiver id. One caregiver performs only one round in their working day, each round consists of several steps. Each step represents one care service operated by this caregiver, with the characteristics such as care service's start time, end time, duration, and the travel time to next location. The round of a caregiver denotes the caregiver start his working day from the HHC structure and finish her/his assigned care service at the HHC structure.

- The second output contains the performance about an optimal solution, whose objective function is maximum over several iterations. The information including the 
objective function value, computing time and the value of its 5 components: 1) feasibility: presenting if all the care services are assigned correctly, the value is equal to the total number of the care services in a use case. 2) patients' waiting time satisfaction: checking if the scheduled time for each care service is correctly included in the time window requested by the patients, the maximum value of this part equal to the number of all patients. 3) patients' interoperation satisfaction: similar to 2), it aims at checking the patient's satisfaction in terms of interoperation between the successive requested care services, the maximum value is the same as 2). 4) caregivers' overtime satisfaction: the caregivers' total satisfaction about over working time regarding their legal workload (10h max/day in our case), the maximum value equals the number of all caregivers. 5) caregiver's difficulty balance: the difficulty balancing indicator with respect to the rounds performed by all caregivers. The difficulty of each round is the sum of the care services' weight indicator that performed by a caregiver. The maximum value is approximated to the number of all caregivers.

\section{Visualiser}

This generator transforms a daily planning result in .csv format into a .json format adapted to AmCharts, a JavaScript library. This library was chosen mainly for its visual aesthetics and implementation flexibility. Different types of charts and axes can easily be manipulated. For example, Bar Charts (Column Series) can be used with nontraditional axes such as Date Axes to create a unique visualization that answers specific needs. Being able to combine axes and chart types as needed is powerful because unique visualizations can be achieved. One way of visualizing a caregiver's round is through a Gantt Chart. Traditionally, these charts are task oriented: each line represents a specific task. In our case, it is more interesting to create a caregiver-oriented chart. This gives an effortless visualization of one's round for a workday. The library's flexibility allows the use of four main elements to answer our needs straightforwardly: Chart Data (.json), a Date Axis (timeline), a Category Axis (caregivers) and a Column Data Series (care services). Once the .csv data is converted to an appropriate .json format through a JavaScript parsing program, and the data is bound to the series correctly, a Gantt Chart is generated.

The JSON data is composed of two types of objects: care service objects, Itinerary Time objects. A JSON object represents a task that will be placed in the Gantt Chart. Task information such as the name of the caregiver, the starting time, the ending time, the duration, the complete title, the difficulty of a care service, the patient concerned by a care service and a color associated to the task. From this formatted data, multiple views of the Gantt Chart have been developed.

The basic view is a simple color code: different colors with the legend for a care service requested by a patient, orange for an itinerary. The care service id is written on its associated task so that the care service is rapidly identified. When the user hovers over a task, a pop up appears. Inside this popup are the following elements:

- The complete title of the task, for clarity.

- The starting time and ending time. This way, the user can get a precise time rapidly without needing an overcrowded Date Axis.

- The duration of the task, so the user does not have to calculate the duration.

In the case of a care service task, there is also:

- The difficulty of the care service, so the user can estimate what effort needs to be put into a care service.

- The patient concerned by the care service.

The Gantt Chart visualization is shown in Fig. 5. The objective is to visualize the result of rounds through a graphic and then, in a second step, to fix the useful indicators to identify the quality of a solution thus presented. To clarify the example shown in Fig. 5, the result show the Gantt chart visualization regarding the solution generated by TS for a use case with 13 care service requested by 6 patients and 3 available caregivers. The daily working round for each caregiver is therefore:

- caregiver 11: 143 61>89 85

- caregiver 41:20>54 $21>50$

- caregiver $50: 16$ 17 23 - 144

The light orange zone shows the itinerary time between the living place of the patients. The color of the care services represents their requestors. For example, there is no travel time between the service 16 and 17 as these 2 services are requested by the same patient (patient 11). The pop-up shows the complete information for the assigned care service such as

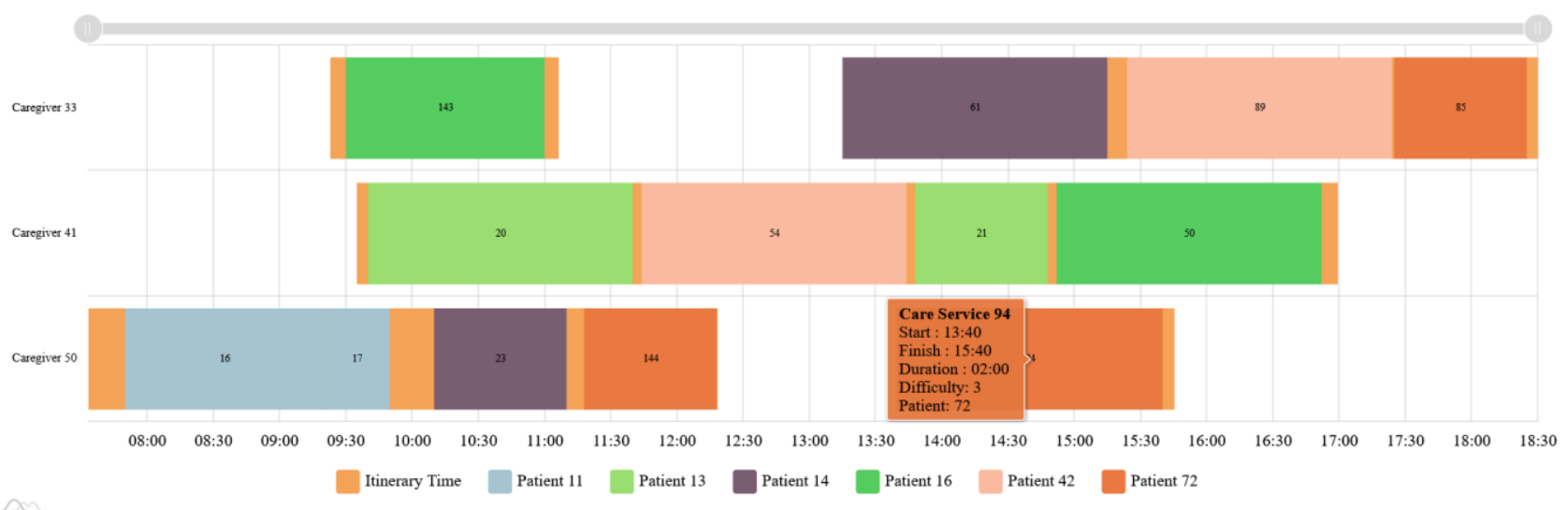

Fig. 5 Graphical representation under Gantt chart for the solution of a use case: 13 care services performed by 3 caregivers 
the planned schedule, duration, difficulty indicator and the requestor.

\section{CONCLUSION AND RESEARCH PERSPECTIVES}

This research work proposes a decision-making support platform named BLPAD.Core to solve the HHCSRP systematically based on the solution approach and benchmark studies. 3 modules included in BLPAD.Core : 1) Based on the real dataset, case generator is to create a standard structure for feeding our own metaheuristic oriented mathematical formulation, by inspiring the similar open access benchmark among the literature. 2) An optimizer embedding two popular metaheuristics for generating the optimum solutions, which will also allow us to assess comparatively the quality of the solutions generated by these two algorithms in a next step. 3) a visualizer which provides a comprehensive view regarding the solution to the decision maker.

In the future, by using BLPAD.Core, this environment will enable us to draw conclusions on the comparison of the 2 metaheuristics from experimental studies. Then, an indicatorsoriented dashboard will be developed to provide the decision maker an evaluation platform regarding the different optimal solutions. In addition, we wish to enrich our platform by developing an exact method or another approach such as multi-agent systems, to invest the comparisons field between the different resolution approaches in a near-real simulated environment or even a real-environment.

\section{REFERENCES}

[1] C. Fikar and P. Hirsch, "Home health care routing and scheduling: A review," Computers \& Operations Research, vol. 77, pp. 86-95, Jan. 2017, doi: 10.1016/j.cor.2016.07.019.

[2] M. Cissé, S. Yalçındağ, Y. Kergosien, E. Şahin, C. Lenté, and A. Matta, "OR problems related to Home Health Care: A review of relevant routing and scheduling problems," Operations Research for Health Care, vol. 13-14, no. Supplement C, pp. 1-22, Jun. 2017, doi: 10.1016/j.orhc.2017.06.001.

[3] M. Di Mascolo, M.-L. Espinouse, and C. E. Ozkan, "Synchronization between human resources in Home Health Care context," in Proceedings of the International Conference on Health Care Systems Engineering, 2014, pp. 73-86.

[4] M. A. Masmoudi and N. Cheikhrouhou, "Heterogeneous Vehicle Routing Problems with Synchronization: Application to Homecare Scheduling Routing Problem," presented at the GISEH 2018, Aug. 2018.

[5] L. Xiao, M. Dridi, and A. H. El Hassani, "Mathematical model for the home health care scheduling and routing problem with flexible lunch break requirements," IFAC-PapersOnLine, vol. 51, no. 11, pp. 334$339,2018$.

[6] R. B. Bachouch, A. Guinet, and S. Hajri-Gabouj, "A decision-making tool for home health care nurses' planning," in Supply Chain Forum: an International Journal, 2011, vol. 12, no. 1, pp. 14-20.

[7] A. F. Torres-Ramos, E. H. Alfonso-Lizarazo, L. S. Reyes-Rubiano, and C. L. Quintero-Araújo, "Mathematical Model for the Home Health Care Routing and Scheduling Problem with Multiple Treatments and Time Windows," Mathematical Methods in Science and Engineering, p. 7.

[8] J. Decerle, O. Grunder, A. Hajjam El Hassani, and O. Barakat, "A memetic algorithm for a home health care routing and scheduling problem," Operations Research for Health Care, vol. 16, pp. 59-71, Mar. 2018, doi: 10.1016/j.orhc.2018.01.004.

[9] C.-C. Lin, L.-P. Hung, W.-Y. Liu, and M.-C. Tsai, "Jointly rostering, routing, and rerostering for home health care services: A harmony search approach with genetic, saturation, inheritance, and immigrant schemes," Computers \& Industrial Engineering, vol. 115, pp. 151166, Jan. 2018, doi: 10.1016/j.cie.2017.11.004.
[10] A. Hertz and N. Lahrichi, "A patient assignment algorithm for home care services," Journal of the Operational Research Society, vol. 60, no. 4, pp. 481-495, 2009.

[11] K.-D. Rest and P. Hirsch, "Daily scheduling of home health care services using time-dependent public transport," Flex Serv Manuf J, vol. 28, no. 3, pp. 495-525, Sep. 2016, doi: 10.1007/s10696-015-92271.

[12] R. Liu, X. Xie, and T. Garaix, "Hybridization of tabu search with feasible and infeasible local searches for periodic home health care logistics," Omega, vol. 47, pp. 17-32, Sep. 2014, doi: 10.1016/j.omega.2014.03.003.

[13] N. Triki, T. Garaix, and X. Xie, "A two-phase approach for periodic home health care planning," in 2014 IEEE International Conference on Automation Science and Engineering (CASE), Aug. 2014, pp. 518523, doi: 10.1109/CoASE.2014.6899375.

[14] N. Szander, L. Ros-McDonnell, and M. V. de la Fuente, "Algorithm for Efficient and Sustainable Home Health Care Delivery Scheduling," in New Global Perspectives on Industrial Engineering and Management, 2019, pp. 315-323.

[15] Y. Shi, T. Boudouh, and O. Grunder, "A robust optimization for a home health care routing and scheduling problem with consideration of uncertain travel and service times," Transportation Research Part E: Logistics and Transportation Review, vol. 128, pp. 52-95, Aug. 2019, doi: 10.1016/j.tre.2019.05.015.

[16] B. Yuan and Z. Jiang, "Disruption Management for the Real-Time Home Caregiver Scheduling and Routing Problem," Sustainability, vol. 9, no. 12, p. 2178, Nov. 2017, doi: 10.3390/su9122178.

[17] N. Manavizadeh, H. Farrokhi-Asl, and P. Beiraghdar, "Using a metaheuristic algorithm for solving a home health care routing and scheduling problem," Journal of Project Management, vol. 5, no. 1, pp. 27-40, 2020.

[18] K. Braekers, R. F. Hartl, S. N. Parragh, and F. Tricoire, "A bi-objective home care scheduling problem: Analyzing the trade-off between costs and client inconvenience," European Journal of Operational Research, vol. 248, no. 2, pp. 428-443, Jan. 2016, doi: 10.1016/j.ejor.2015.07.028

[19] Y. Shi, T. Boudouh, O. Grunder, and D. Wang, "Modeling and Solving Simultaneous Delivery and Pick-up problem with Stochastic Travel and Service Times in Home Health Care," Expert Systems with Applications, Feb. 2018, doi: 10.1016/j.eswa.2018.02.025.

[20] C. He and Y. Shi, "Workforce Scheduling and Routing for Home Health Care Services," in Proceedings of the 3rd International Conference on Computer Science and Application Engineering, 2019, pp. $1-5$.

[21] G. Hiermann, M. Prandtstetter, A. Rendl, J. Puchinger, and G. R. Raidl, "Metaheuristics for solving a multimodal home-healthcare scheduling problem," Cent Eur J Oper Res, vol. 23, no. 1, pp. 89-113, Mar. 2015, doi: 10.1007/s10100-013-0305-8.

[22] S. Sarmady, "An Investigation on Tabu Search Parameters," p. 11.

[23] W. Ben-Ameur, "Computing the Initial Temperature of Simulated Annealing," Comp. Opt. and Appl., vol. 29, pp. 369-385, 2004, doi: 10.1023/B:COAP.0000044187.23143.bd.

[24] D. Bredström and M. Rönnqvist, "Combined vehicle routing and scheduling with temporal precedence and synchronization constraints," European Journal of Operational Research, vol. 191, no. 1, pp. 19-31, Nov. 2008, doi: 10.1016/j.ejor.2007.07.033.

[25] D. S. Mankowska, F. Meisel, and C. Bierwirth, "The home health care routing and scheduling problem with interdependent services," Health Care Manag Sci, vol. 17, no. 1, pp. 15-30, Mar. 2014, doi: 10.1007/s10729-013-9243-1.

[26] L. Zhang et al., "Home Health Care Daily Planning Considering the Satisfaction of all the Stakeholders," in 2019 International Conference on Industrial Engineering and Systems Management (IESM), Sep. 2019, pp. 1-6, doi: 10.1109/IESM45758.2019.8948201.

[27] L. Zhang, E. Lamine, F. Fontanili, C. Bortolaso, M. Derras, and H. Pingaud, "A Decision-Making Support System for Operational Coordination of Home Health Care Services," in 2019 IEEE/ACS 16th International Conference on Computer Systems and Applications (AICCSA), Nov. 2019, pp. 1-6, doi: 10.1109/AICCSA47632.2019.9035219.

[28] J. Silberholz and B. Golden, "Comparison of Metaheuristics," in Handbook of Metaheuristics, M. Gendreau and J.-Y. Potvin, Eds. Boston, MA: Springer US, 2010, pp. 625-640. 